\title{
The nutrition, cultivation and biotechnology of Stropharia rugosoannulata
}

\author{
Hu $Y^{1,2,3}$, Kakumyan $P^{2}$, Bandara AR ${ }^{1}$, Mortimer $\mathrm{PE}^{1 *}$ \\ ${ }^{1}$ Centre for Mountain Futures, Kunming Institute of Botany, Chinese Academy of Sciences, Kunming 650201, Yunnan, \\ China \\ ${ }^{2}$ School of Science, Mae Fah Luang University, Chiang Rai 57100, Thailand \\ ${ }^{3}$ Center of Excellence in Fungal Research, Mae Fah Luang University, Chiang Rai 57100, Thailand
}

Hu Y, Kakumyan P, Bandara AR, Mortimer PE 2021 - The nutrition, cultivation and biotechnology of Stropharia rugosoannulata. Fungal Biotec 1(1), 13-25, Doi 10.5943/FunBiotec/1/1/2

\begin{abstract}
Stropharia rugosoannulata contain polysaccharides, sterols, lectins, flavonoids, vitamins and minerals. Various bioactivities are reported from this mushroom, such as antidiabetic, antimicrobial, antioxidant, antiproliferative, antitumor, immunomodulatory and suppressing osteoclast formation properties. The mushroom has also been cultivated to increase rural and urban incomes and remediate soils. Soils contaminated by organic pollutants have been remediated by fungal extracellular enzymes, such as laccase and manganese peroxidase. The present review summarizes the nutritional value of the mushroom, bioactive compounds, cultivation methods and biotechnology. This review also provides suggestions for its future applications.
\end{abstract}

Keywords - agroforestry - bioactive compounds - bioremediation - field cultivation - fungi

\section{Introduction}

Stropharia rugosoannulata Farl. ex Murrill is commonly known as the Wine-cap mushroom, King Stropharia, Winter mushroom, Garden giant, or Burgundy mushroom (Yang et al. 2021). It is an agaric mushroom of Strophariaceae, which is distributed across northern temperature zones (Suzuki et al. 2019). Basidiomes are terrestrial, solitary or gregarious and can be found on humusand nitrogen-rich soils, particularly in gardens, parks, grasslands and can even grow on manure. Stropharia rugosoannulata is edible, cultivated for household consumption (Pegler 2001, Bruhn et al. 2010) and grown commercially (He et al. 2012). The Food and Agriculture Organization (FAO) recommends the consumption of this mushroom because it can help mitigate a variety of cancerrelated illnesses (de Oliveira et al. 2020). Research performed on S. rugosoannulata has revealed applications of this fungus as a functional and medical food via suppressing osteoclast formation as well as having antidiabetic, antimicrobial, antioxidant, antiproliferative, antitumor and immunomodulatory properties (He et al. 2012, Zhai et al. 2013, Zhang et al. 2016, Liu et al. 2020).

Stropharia rugosoannulata was first domesticated in 1969 in Germany, followed by Poland, the former Czechoslovakia (Czech Republic or Slovakia), Hungary and the former Soviet Union (Stamets \& Chilton 1983, Sharma et al. 2007). By 1989, the annual commercial production of $S$. rugosoannulata in Europe reached approximately 1300 tons (Domondon et al. 2000). China started the cultivation industry of S. rugosoannulata in 1990 (Wan \& Bau 2005, Yan et al. 2020). By 2019, the cultivation area in China had reached approximately 1300 hectares, with a production of about 
30-45 tons per hectare, and the export volume reached 3,000 tons in 2018 (CEMBN 2019). It is currently cultivated at large scale in many provinces in China, such as Hebei, Shandong, Yunnan and Zhejiang (Tian et al. 2002, Jin et al. 2020, Hua et al. 2021).

Traditional cultivation of $S$. rugosoannulata occurs from September to April of the second year, featuring an in-depth management strategy. It is often cultivated under economic forests or orchards in field conditions. The main steps include spawn production, composting, bed preparation, inoculation, management and harvest, with optimal temperatures and humidity between $20-30^{\circ} \mathrm{C}$ and $70-85 \%$, respectively (Bruhn et al. 2010). This mushroom can be cultivated with various kinds of raw materials, such as cereal straw, sawdust, sugarcane, rice husk, cottonseed hull and corncobs (Bao 2015). Cereal straw such as paddy, corn and soybean are widely used to cultivate S. rugosoannulata (Szudyga 1978, Yu et al. 2014). Wood chips of Alnus, Hevea, Populus, Quercus, Tilia have been reported to produce considerable $S$. rugosoannulata yields (Domondon et al. 2000, Stamets 2011), and a yield of around $4 \mathrm{~kg} / \mathrm{m}^{2}$ was reported when using Hevea sawdust as a growing substrate (Huang et al. 2019). However, wood chips from trees like Salix contain a high amount of salicylic acid and are not suitable for the cultivation of $S$. rugosoannulata because this compound suppresses the growth of fungal mycelium (Raskin 1992). After all the beds are well colonized by fungal mycelium, the substrate is overlaid with a $3-5 \mathrm{~cm}$ deep soil casing layer. The casing layers are kept humid in case the substrates lose water. It takes 40-60 days for mushrooms to form after placing the soil casing layer. In general, the raw materials and climatic conditions for large-scale cultivation of $S$. rugosoannulata are critical, since farmers could reduce costs with cheap raw materials and increase yields with suitable climatic conditions, improving the economic performance of their land.

Fungal biotechnology can play a crucial role in agroforestry systems, capable of producing resilient sources of food, feed, chemicals, fuels and materials (Meyer et al. 2020). In addition, the cultivation of $S$. rugosoannulata supplements local income streams through building a more efficient circular economy while also amending and remediating soils for sustainable landscape management (Fig. 1). This review summarizes current knowledge of nutrients, cultivation, and biotechnology of $S$. rugosoannulata. Its roles in circular economies and bioremediation are discussed, as they could be helpful for the full utilization of this mushroom.
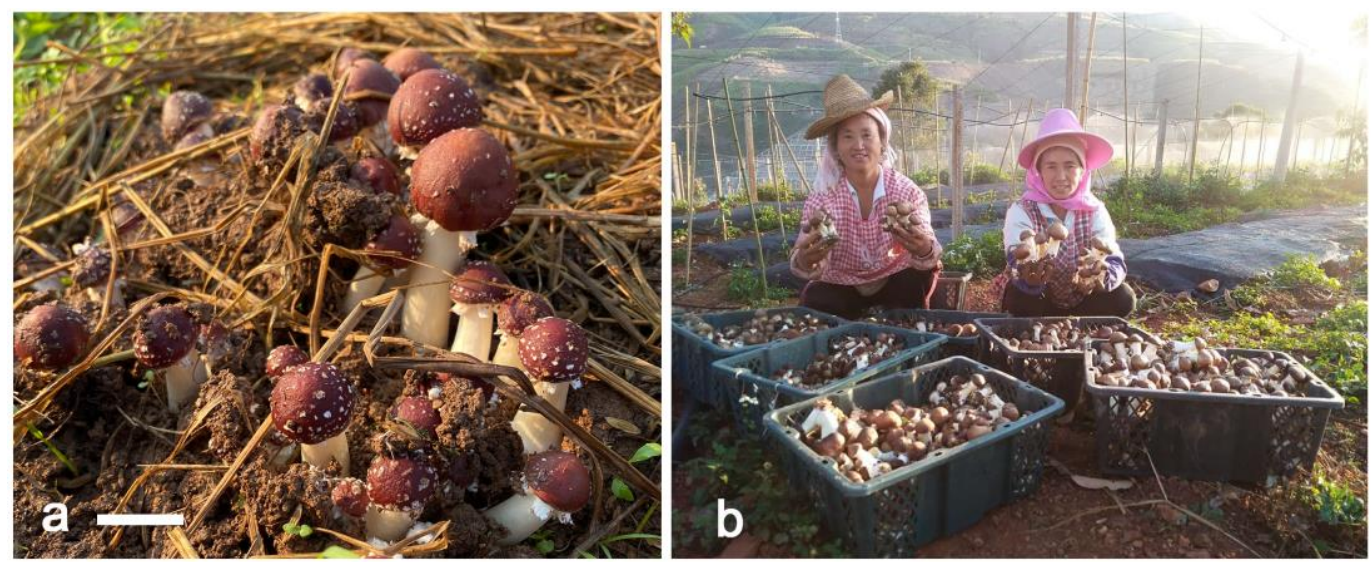

Fig. 1 - Cultivation of Stropharia rugosoannulata supplements local income in Yunnan, China. Scale bar: $5 \mathrm{~cm}$. Photo credit: Hu Y.

\section{Food resources and nutritional value}

Stropharia rugosoannulata basidiomes contain abundant macro- and micro-nutrients. The nutritional components of $S$. rugosoannulata have been described in previous studies (Liu et al. 2012, 2019a), with the main components featuring ash, protein, crude fat, carbohydrates, minerals, coarse cellulose, energy, vitamins, fatty acids and amino acids. Table 1 lists the composition of macronutrients and minerals in the basidiomes of $S$. rugosoannulata and compares the composition with Agaricus bisporus, Lentinula edodes and Pleurotus ostreatus, which are some of the most 
popular and common edible mushroom species. Based on the data from Table 1, the ash content of wild and cultivated S. rugosoannulata (6.04-8.72\%) is higher than L. edodes (4.29-6.24\%), while no significant difference existed between A. bisporus (6.04-9.74\%) and P. ostreatus (7.62-9.65\%). It is clear that basidiomes from both wild and cultivated $S$. rugosoannulata contain a high amount of crude protein (25.75-25.89\%) when compared with A. bisporus (3.05-14.08\%), L. edodes (12.76-16.00\%) and P. ostreatus (18.35-25.68\%). The crude fat content of wild and cultivated $S$. rugosoannulata $(2.19-3.72 \%)$ is higher than L. edodes $(1.01-1.14 \%)$, wild P. ostreatus $(2.08 \%)$ and cultivated A. bisporus (1.78\%), while being lower than wild A. bisporus $(7.18 \%)$. The carbohydrates and fibers content of S. rugosoannulata (61.91-64.35\%) is lower than A. bisporus (74.00-89.13\%), L. edodes (76.62-81.94\%), and P. ostreatus (62.54-71.26\%). Moreover, the micronutrient mineral content of $S$. rugosoannulata is abundant compared to A. bisporus, L. edodes and $P$. ostreatus. For example, $S$. rugosoannulata basidiomes contain abundant Fe (195.00-244.10 $\mu \mathrm{g} / \mathrm{g}$, dry weight), Mn (40.60-59.00 $\mu \mathrm{g} / \mathrm{g}$, dry weight) and $\mathrm{Cu}(16.00-29.00 \mu \mathrm{g} / \mathrm{g}$, dry weight), reaching higher levels than the other three species (Table 1). The $\mathrm{Zn}$ content of $S$. rugosoannulata (54.40-102.00 $\mu \mathrm{g} / \mathrm{g}$, dry weight) is higher than A. bisporus $(17.80-22.98 \mu \mathrm{g} / \mathrm{g}$, dry weight) and $P$. ostreatus (29.80-42.83 $\mu \mathrm{g} / \mathrm{g}$, dry weight), while being similar to L. edodes (53.82-94.40 $\mu \mathrm{g} / \mathrm{g}$, dry weight). From the above data, it can be concluded that both wild and cultivated $S$. rugosoannulata are prized sources of nutrients owing to high amounts of protein, crude fat and micronutrient minerals.

\section{Bioactive compounds}

Stropharia rugosoannulata contains promising bioactive compounds in both its fungal mycelia and basidiomes. The main bioactive components of this mushroom include monosaccharides, polysaccharides, sterols, lectins, flavonoids and phenols, some of them have the ability of antioxidant, antibacterial, antitumor and antidiabetic effects (Wang et al. 2018, Liu et al. 2020). The main bioactive components in the basidiomes are monosaccharides and polysaccharides, which vary in their properties based on extraction methods and different parts of basidiomes (Wang et al. 2018). Monosaccharides including mannose, ribose, glucuronic acid, glucose and galactose were detected from fresh basidiomes of $S$. rugosoannulata, and glucose was the main monosaccharide (Liu et al. 2020). The monosaccharide composition included galactose and glucose with a 3:1 ratio in fresh $S$. rugosoannulata (Jiang et al. 2020). In addition, Jiang et al. (2020) also isolated a new polysaccharide from S. rugosoannulata and showed this polysaccharide stimulates immunological activities such as the growth of $\mathrm{T}$ lymphocytes, B lymphocytes and RAW264.7 cells as well as the secretion of $\operatorname{IgA}$, IgD, and IgG by B lymphocytes. The main two types of polysaccharides and antioxidant characterizations from $S$. rugosoannulata were studied by Liu et al. (2020). $\alpha$-type and $\beta$-type glycosidic linkages were detected in one kind of polysaccharide, while the $\beta$-constitution dominated in the other polysaccharide; moreover, both polysaccharides showed potential antioxidant activities on $\mathrm{ABTS}^{+}$, DPPH and $\mathrm{OH}^{-}$radicals $(63.29-$ $100 \%, 46.67-62.50 \%$ and 39.28-98.59\%, respectively). Exopolysaccharide production from pure fungal mycelium of $S$. rugosoannulata was investigated by Zhou et al. (2010), and the authors concluded that the optimized conditions for the cultivation temperature, time and volume of media for exopolysaccharide production were around $28^{\circ} \mathrm{C}, 6-7$ days and $110 \mathrm{ml}$, respectively. Under optimal conditions, the maximal production of exopolysaccharide and dry mycelium weight was $1.50 \mathrm{gl}^{-1}$ and $9.88 \mathrm{gl}^{-1}$, respectively. An exopolysaccharide produced by submerged culture of $S$. rugosoannulata was studied by Zhai et al. (2013), and the maximum dry mycelial biomass (around $16.35 \mathrm{gl}^{-1}$ ) was detected after five days of incubation. Exopolysaccharide production reached its maximal level (10.83 $\left.\mathrm{gl}^{-1}\right)$ after eight days of incubation. The hypoglycemic effect of exopolysaccharides was studied on diabetic rats, and the results showed some promising effects of exopolysaccharides on hyperglycemia prevention in diabetic patients (Zhai et al. 2013). 
Table 1. Macronutrients and mineral composition in both wild and cultivated Stropharia rugosoannulata, and compared with Agaricus bisporus, Lentinula edodes and Pleurotus ostreatus.

\begin{tabular}{|c|c|c|c|c|c|c|c|c|c|c|c|}
\hline Species & $\begin{array}{l}\text { Growing } \\
\text { condition }\end{array}$ & $\begin{array}{l}\text { Ash } \\
(\%)\end{array}$ & $\begin{array}{l}\text { Crude } \\
\text { protein } \\
(\%)\end{array}$ & $\begin{array}{l}\text { Crude } \\
\text { fat }(\%)\end{array}$ & $\begin{array}{l}\text { Carbohydrates } \\
\text { and fibers }(\%)\end{array}$ & $\begin{array}{c}\mathrm{Fe} \\
(\mu \mathrm{g} / \mathrm{g} \\
\mathrm{dry} \\
\text { weight })\end{array}$ & $\begin{array}{c}\mathrm{Zn}(\mu \mathrm{g} / \mathrm{g}, \\
\text { dry } \\
\text { weight })\end{array}$ & $\begin{array}{c}\mathrm{Ca}(\mu \mathrm{g} / \mathrm{g}, \\
\text { dry } \\
\text { weight })\end{array}$ & $\begin{array}{l}\text { Mn }(\mu \mathrm{g} / \mathrm{g}, \\
\text { dry } \\
\text { weight })\end{array}$ & $\begin{array}{c}\mathrm{Cu}(\mu \mathrm{g} / \mathrm{g}, \\
\text { dry } \\
\text { weight })\end{array}$ & References \\
\hline Stropharia & Wild & 6.04 & 25.89 & 3.72 & 64.35 & 195.00 & 102.00 & $1,371.00$ & 59.00 & 29.00 & Liu et al. (2012) \\
\hline rugosoannulata & Cultivated & 8.72 & 25.75 & 2.19 & 61.91 & 244.10 & 54.40 & 151.90 & 40.60 & 16.00 & Wang (2007) \\
\hline \multirow[t]{2}{*}{ Agaricus bisporus } & Wild & 9.74 & 14.08 & 7.18 & 74.00 & 126.00 & 17.80 & 74.50 & 22.30 & 5.22 & $\begin{array}{l}\text { Demirbaş (2001), } \\
\text { Jedidi et al. (2017) }\end{array}$ \\
\hline & Cultivated & 6.04 & 3.05 & 1.78 & 89.13 & 21.36 & 22.98 & nd & 4.16 & 16.40 & $\begin{array}{l}\text { Owaid (2015), } \\
\text { Stojković et al. } \\
(2014)\end{array}$ \\
\hline \multirow[t]{2}{*}{ Lentinula edodes } & Wild & 4.29 & 12.76 & 1.01 & 81.94 & $\begin{array}{c}41.68- \\
60.62\end{array}$ & $\begin{array}{c}53.82- \\
70.72\end{array}$ & $\begin{array}{c}165.00- \\
295.00\end{array}$ & nd & $\begin{array}{l}9.99- \\
13.60\end{array}$ & $\begin{array}{l}\text { Carneiro et al. } \\
\text { (2013), Guo et al. } \\
(2010)\end{array}$ \\
\hline & Cultivated & 6.24 & 16.00 & 1.14 & 76.62 & 148.00 & 94.40 & $1,749.00$ & 10.00 & 14.80 & $\begin{array}{l}\text { Heleno et al. } \\
\text { (2015), } \\
\text { Mallikarjuna et al. } \\
\text { (2012) }\end{array}$ \\
\hline \multirow[t]{2}{*}{$\begin{array}{l}\text { Pleurotus } \\
\text { ostreatus }\end{array}$} & Wild & 7.62 & 24.90 & 2.08 & 65.40 & 86.10 & 29.80 & 106.00 & 6.27 & 13.60 & $\begin{array}{l}\text { Demirbaş (2001), } \\
\text { Beluhan \& } \\
\text { Ranogajec (2011) }\end{array}$ \\
\hline & Cultivated & $\begin{array}{c}7.82- \\
9.65\end{array}$ & $\begin{array}{l}18.35- \\
25.68\end{array}$ & $\begin{array}{c}2.34- \\
2.58\end{array}$ & $62.54-71.26$ & $\begin{array}{l}80.18- \\
129.88\end{array}$ & $\begin{array}{c}42.83- \\
52.10\end{array}$ & $\begin{array}{c}391.00- \\
821.00\end{array}$ & $\begin{array}{c}3.09- \\
7.44\end{array}$ & $\begin{array}{l}9.67- \\
14.60\end{array}$ & Jin et al. (2018) \\
\hline
\end{tabular}

nd, not determined. 
Aside from polysaccharides, S. rugosoannulata contains sterols, lectins, flavonoids and other compounds that constitute active ingredients of edible and medicinal mushrooms. The functional constituents of sterols in S. rugosoannulata were reported by Wu et al. (2011), with five sterols isolated from basidiomes of $S$. rugosoannulata showing anti-fungal activity, inhibition of osteoclasts formation or thapsigargin toxicity. Wu et al. (2012, 2013a, b) extracted particular steroids from the basidiomes of $S$. rugosoannulata by ethyl alcohol and acetone, sterols A-D from S. rugosoannulata were obtained from fresh basidiomes, and a distinct sterol with an unprecedented ether ring from this mushroom was isolated. Sterol A may protect neuronal cells and showed weak anti-Methicillin-resistant Staphylococcus aureus activity (Wu et al. 2012). Zhang et al. (2014) isolated a type of novel lectin with a unique $\mathrm{N}$-terminal sequence from the basidiomes of $S$. rugosoannulata, representing the first purified protein from this mushroom species, and this lectin shows antiproliferative activity toward cancer cells and anti-HIV reverse transcriptase activity compared with lectins isolated from other mushrooms such as A. bisporus, Cyclocybe aegerita and Coprinopsis cinerea. Yan et al. (2020) investigated the basidiomes of $S$. rugosoannulata, leading to a total of sixteen compounds being isolated and identified, including six types of steroids, one type of steroidal saponins, three types of fatty acids, one type of alkane, one type of ceramide, one type of ester, one type of pyrimidine, one type of vitamins and one type of flavonoid. However, the functional activities of these compounds remain unclear and is in need of further study. Based on the existence of potentially undiscovered bioactive compounds, future studies could focus on the mechanisms and actions of the components in S. rugosoannulata that benefit human health and could be developed into functional chemical components for foods or used in the prevention and treatment of chronic diseases.

\section{Cultivation methods of Stropharia rugosoannulata Culture}

Solid and liquid culture media can both be used in the cultivation of S. rugosoannulata. Solid culture media is often prepared with potato dextrose agar, malt extract agar or yeast mannitol agar (Zhang et al. 2005, Nie et al. 2016). Another solid medium uses agricultural waste as the main component, such as wheat straw (Yan et al. 2001). Liquid culture media are often prepared with potato dextrose broth and glucose-based broth media (Wang \& Li 2008). After the media are inoculated via tissue isolation of basidiomes or culture collection, cultures are incubated for mycelium growth. Optimal incubation conditions for the solid culture of $S$. rugosoannulata include $25-30^{\circ} \mathrm{C}$, pH 5-6 and a water content of $75 \%$ for $10-30$ days (Yan et al. 2001). The optimal incubation conditions for liquid culture include $25^{\circ} \mathrm{C}, \mathrm{pH} 6$ and a rotation speed of $120 \mathrm{rpm}$ for 12 days (Liu et al. 2019b).

\section{Spawn production}

Spawn production for commercial cultivation of S. rugosoannulata includes cereal-based spawn and agricultural waste-based spawn; the former type is applied for small-scale cultivation, and the latter is used for large-scale cultivation. Cereal-based spawns are prepared with wellcooked wheat or rice with a $\mathrm{pH} \mathrm{6.5-7}$ and 65-70\% water content ( $\mathrm{Li}$ et al. 2012). Agricultural waste-based spawns are mainly prepared using corn wastes, rice straw or soybean straw (Sun 2013). Sawdust-based spawns are mainly used for large-scale $S$. rugosoannulata cultivation (Li et al. 2013). Spawn bags or bottles should be sterilized at $121^{\circ} \mathrm{C}$ and $105 \mathrm{kpa}$ for 2 hours before inoculation of solid or liquid culture, and the optimal temperature for incubation is $25^{\circ} \mathrm{C}$ (Sun 2013).

\section{Compost preparation}

The materials used for compost preparation come from various agricultural wastes, such as cereal straw, wood sawdust, wheat bran and rice bran (She et al. 2007). Typical formulas for composting include the following:

i. Mulberry branch sawdust 100\% (Li et al. 2012); 
ii. $\quad$ Rice straw 50\%, rice husk 50\% (Chen et al. 2010);

iii. Rice husk 49\%, wood sawdust 49\%, gypsum 1\%, phosphate 1\% (Jiang et al. 2017); and

iv. Spent mushroom substrate $40 \%$, rice husk powder $18 \%$, wood sawdust $20 \%$, cottonseed hull $10 \%$, wheat bran 10\%, gypsum 1\%, sugar $1 \%$ (Jiang et al. 2017).

Before the composting process, all agricultural waste should be ground into pieces approximately $1-3 \mathrm{~cm}$ in diameter. Next, agricultural waste should be soaked until reaching adequate water absorption before draining surplus water. Then, agricultural wastes are mixed and placed in a compost heap, with the dimensions of the compost heap ranging from $1.5 \mathrm{~m}$ in width, $1.5 \mathrm{~m}$ in height and the length is determined by the total amount of compost. After building the heap, the temperature of the heap will increase to $60-70^{\circ} \mathrm{C}$ between the third to fifth day. The compost should be first turned on the eighth day, the second turning should be conducted on the eleventh day and the third and fourth turnings should be conducted on the fourteenth and seventeenth days, respectively, before the compost enters phase II-the pasteurization stage. In this stage, the compost is turned every three days, and after 26 days it is ready for use in cultivation. The optimal water content of compost is 65-75\% (Wan \& Bau 2005). The composting process is briefly summarized in Table 2. The process of composting for the cultivation of $S$. rugosoannulata is similar to Agaricus subrufescens (Wisitrassameewong et al. 2012).

Table 2. Composting process in the cultivation of Stropharia rugosoannulata.

\begin{tabular}{lll}
\hline Calendar & Work content & Compost phase \\
\hline- & Gather the ground $(1-3 \mathrm{~cm})$ raw materials & Preparation \\
Day 0 & Wet the raw materials until adequate water absorption & Temperature increase \\
Day 1 & Mix the compost and build the heap & Composting begins \\
Day 8 & Turn the compost & Phase I \\
Day 11 & Turn the compost & Phase I \\
Day 14 & Turn the compost & Phase I \\
Day 17 & Turn the compost & Phase I ends; phase II begins \\
Day 20 & Turn the compost & Phase II \\
Day 23 & Turn the compost & Phase II \\
Day 26 & Spread and cool the compost in the field & Ready to use for cultivation \\
\hline
\end{tabular}

\section{Cultivation process}

The best sites for the field cultivation of $S$. rugosoannulata are vacant lands, orchards or plantations; the optimal temperature for mushroom growing ranges between $20-30^{\circ} \mathrm{C}$; and the cultivation site should be close to a water source, with good drainage, fertile soil, shelter and protection from strong wind. Beforehand, weeds must be removed, and soil must be flattened. Then, drainages ditches should be dug and land beds arranged using a grid width of 0.8-1.2 meters. Finally, quicklime should be spread on the bed and drainage ditches before beginning spawn inoculation (Bao 2015).

During the inoculation process, a 10-cm-thick first layer of compost should be spread on the bed. Then, the spawn should be inoculated by the dibbling method before spreading one more layer of compost on the bed after both applications. The total compost will be approximately $20-35 \mathrm{~cm}$ thick with 2-3 layers, and a casing soil layer around $2-5 \mathrm{~cm}$ thick will cover the bed. Finally, spreading one layer of quicklime on the casing soil is needed to prevent contamination and provide insect repellant (She et al. 2007). The amounts of compost and spawn should range between 20-25 $\mathrm{kg} / \mathrm{m}^{2}$ (dry weight) with 5\% spawn (w/w) (Tian et al. 2002, Yan \& Jiang 2005). The illustration of S. rugosoannulata cultivation in field conditions is shown in Fig. 2.

\section{Management}

The best temperature range for the mycelial growth of $S$. rugosoannulata in compost is 23 $27^{\circ} \mathrm{C}$; the humidity for compost should be around $70-75 \%$; and the ideal humidity is $90-95 \%$ when entering the fruiting stage (Wan \& Bau 2005). Water content and humidity should be maintained 
through watering the growing lands. Light control in the fields is conducted via black sunshade plastic nets with over $80 \%$ shading coverage or rice straw with over $90 \%$ shading coverage. In practice, it is also possible to place rice straw on the land surface or hang $2 \mathrm{~m}$ above the soil via a sunshade net (Gong et al. 2016). Yellow luring boards are often used for insect control, such as diptera and lepidoptera in fields cultivating S. rugosoannulata (Zhu 2018).

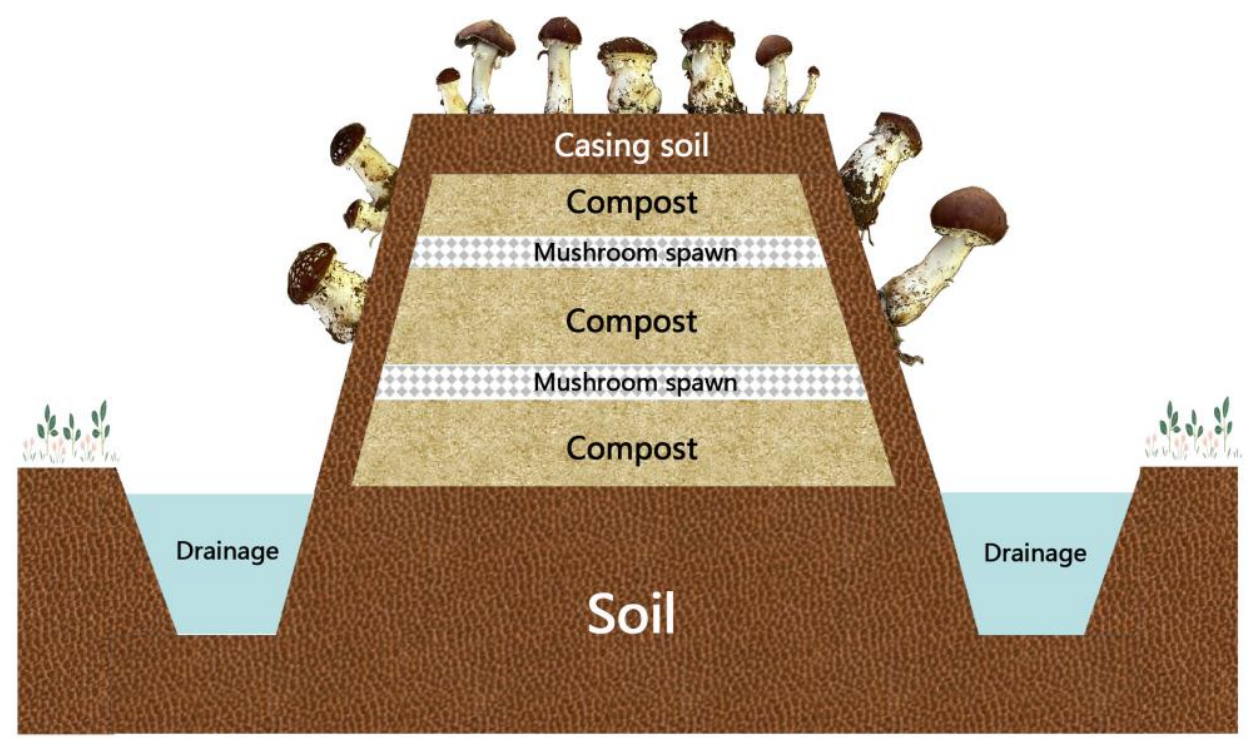

Fig. 2 - Profile of Stropharia rugosoannulata cultivation in the field. Three layers of compost and two layers of mushroom spawn are incubated. Drainage can help bolster and maintain humidity in the system.

\section{Harvest and yield}

After 30-60 days, the first flush of the harvest will appear. Mushroom collection should be done carefully to avoid disturbing the compost and casing soil. After each harvest, the soil remaining at the surface of mushrooms should be cleaned by using a sharp knife and tissue paper. The products should be sold or processed as soon as possible, using techniques like salt processing with a $40 \%$ saturated salt solution or drying in an oven (Wan \& Bau 2005). A total of 3-4 mushroom flushes over 3-4 months can be conducted throughout the cultivation process. Chen et al. (2010) reported $81.74 \%$ biological efficiency when using rice straw $50 \%$ and rice husk $50 \%$ as compost formula, which is higher than rice straw $100 \%$ (biological efficiency $44.04 \%$ ) and rice husk $100 \%$ (biological efficiency $56.72 \%$ ). Gong et al. (2016) reported a yield of $5.325 \mathrm{~kg} / \mathrm{m}^{2}$ using $50 \%$ rice husk, $30 \%$ corn cobs and $20 \%$ sawdust as the compost formula. Bao (2015) reported the optimal formula is sawdust $68.2 \%$, wheat straw $20.3 \%$, and corn cobs $11.5 \%$, which yielded 4.71 $\mathrm{kg} / \mathrm{m}^{2}$. Zhang et al. (2017) reported a yield of $6.98 \mathrm{~kg} / \mathrm{m}^{2}$ and biological efficiency of $52.3 \%$ using spent mushroom substrate $40 \%$, corn cobs $25 \%$, soybean straw $25 \%$, rice bran $8 \%$ and calcium oxide $2 \%$ as the compost formula.

\section{Agroforestry circular economy}

Cultivation of $S$. rugosoannulata mushroom has become a standard forest-farming practice in agroforestry. This mushroom is produced on lignocellulosic materials such as wood sawdust, cereal straw and seed husk, and low-quality agricultural waste can be converted into high-quality food (Bruhn et al. 2010, Hu \& Zhang 2013). Spent mushroom substrates left over after harvesting basidiomes contain a high level of organic matter, nitrogen, phosphorus, potassium and other nutrients, which are byproducts of edible mushroom cultivation. Spent mushroom substrates obtained after mushroom cultivation are thus reused as a resource for other agricultural products, such as fertilizer for seedling growth, animal feed and the growing substrate of other mushroom 
species (Hyde et al. 2019, Meyer et al. 2020). The outdoor cultivation of S. rugosoannulata allows for its agricultural production to be more efficient and sustainable. Duan et al. (2018) conducted $S$. rugosoannulata undergrowth cultivation across eight different forest types in Kunming, China, and results showed a significant correlation between mushroom yield and forest canopy. Thus, $S$. rugosoannulata cultivation benefits agro-food production, and at the same time, growing mushrooms under planted forests such as apple trees (Malus Mill), waxberry (Myrica L.) and walnut trees (Juglans regia L.) increases fruit and biomass yield in artificial forest ecosystems, bolstering economic and ecological benefits. Cultivation of $S$. rugosoannulata has become a staple practice in agroforestry, and there is now a range of applications for $S$. rugosoannulata mushrooms (Bruhn et al. 2010). Two programs run in Honghe and Luliang County, Yunnan Province, China (Fig. 3) assisted local farmers with the cultivation of S. rugosoannulata, planting it with passion fruit (Passiflora edulia) and intercropping with lettuce (Lactuca sativa). The spent mushroom substrates did not produce more mushroom basidiomes while still containing abundant nutrient elements (Gong et al. 2018) that were usable as organic fertilizer for passion fruit and lettuce production. At the same time, the branches and stems of plants can be used as raw materials for compost in the mushroom cultivation process, forming a circular economy.
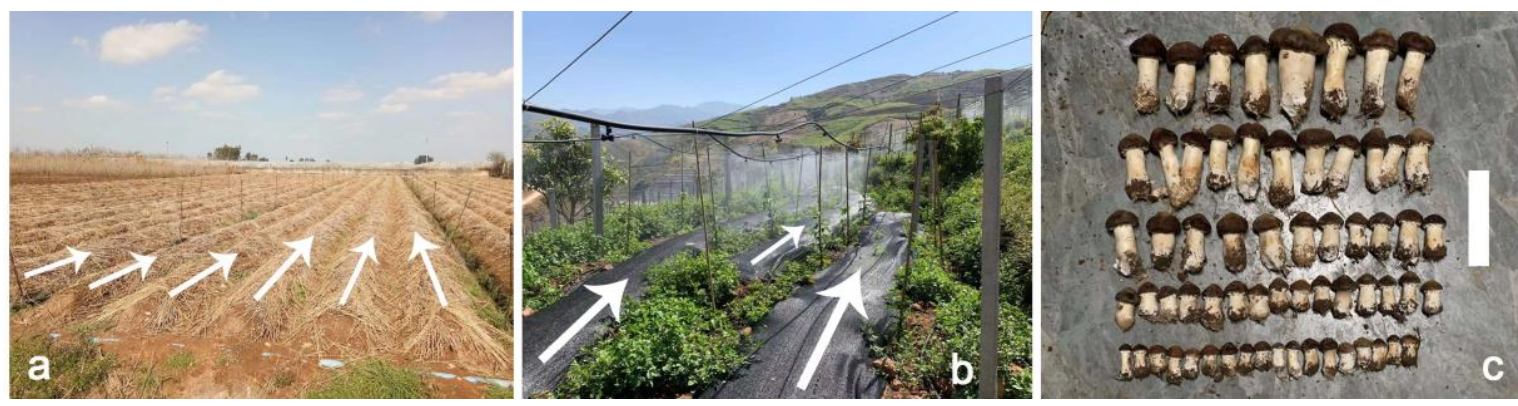

Fig. 3 - Cultivation of Stropharia rugosoannulata. a, intercropping S. rugosoannulata with Lettuce (Lactuca sativa), b, incorporating S. rugosoannulata with Passion fruit (Passiflora edulia), c, grading of fruiting bodies of $S$. rugosoannulata. Arrow: location of cultivation. Scale bar: $15 \mathrm{~cm}$. Photo credit: $\mathrm{Hu}$ Y and Zhu J.

\section{Soil amender and mycoremediation}

During the cultivation of $S$. rugosoannulata, growing substrates cannot be fully utilized by mycelia and transferred to mushroom products. Spent mushroom substrates contain abundant hemicelluloses, cellulose, ash, carbohydrates, protein, calcium, phosphorus potassium and other elements that can improve soil quality in various ways, such as in situ composting and functioning as a soil amender in agricultural, horticulture and animal husbandry fields (Hanafi et al. 2018). Duan et al. (2019) experimented with the cultivation of $S$. rugosoannulata in an under-forest (Cunninghamia lanceolata - Pinus armandii), and results indicated that mushroom cultivation increases soil porosity, organic matter content, hydrolytic nitrogen, available phosphorus and available potassium. Gong et al. (2018) also conducted cultivation experiments of $S$. rugosoannulata. They tested the soil quality and bacterial communities among different farming regimes, with the results showing positive effects on soil physicochemical properties, suggesting that one-year-interval cultivation regimes are potentially the most appropriate system for forest soils. Overall, it is feasible to cultivate $S$. rugosoannulata with a fitting agricultural regimen for soil improvement in food production landscapes.

Since $S$. rugosoannulata can be cultivated in outdoor fields, this saprobic mushroom is a promising candidate for soil bioremediation. Stropharia rugosoannulata has been studied concerning the degradation of pollutants in different environments. Anasonye et al. (2015) showed the potential of $S$. rugosoannulata for degrading 2,4,6-trinitrotoluene (TNT) in soils, a toxic and possible human carcinogen, and the activity of manganese peroxidase and laccase were both detected in the soil. Steffen et al. (2007) and Pozdnyakova et al. (2018) reported that $S$. 
rugosoannulata can degrade polycyclic aromatic hydrocarbons (PAHs), a large group of organic compounds containing two or more aromatic rings with toxic, mutagenic and carcinogenic properties. PAHs were converted into low molecular compounds with non-toxic or hypotoxic soil by S. rugosoannulata. Valentín et al. (2013) and Anasonye et al. (2014) investigated the potential of fungal mycelia to degrade chlorinated phenols, dibenzo- $p$-dioxins and furans on wood and soil in sawmill areas. Stropharia rugosoannulata shows 13-73\% degradation and mineralization of pollutants and proved to be a suitable fungus for soil mycoremediation. In addition to soils, $S$. rugosoannulata also show promising biodegradation ability in contaminated waters: one study (Castellet-Rovira et al. 2018) showed the fungus reaching over 70\% degradation for carbamazepine, a type of pharmaceutically active compound found in contaminated water. In addition, Luo et al. (2006) found $S$. rugosoannulata produces unique spiny cells called acanthocytes, which immobilize and digest the nematode Panagrellus redivivus in soils. Thus, S. rugosoannulata is a promising candidate for bioattenuation and bioremediation processes.

\section{Conclusion and perspectives}

This study reviewed the potential uses of the edible mushroom species $S$. rugosoannulata as a nutritional food resource as well as its bioactive compounds, cultivation, agroforestry circular economy and applications in mycoremediation. Stropharia rugosoannulata contains abundant nutrients and bioactive compounds in mycelium and basidiomes, allowing it to function as an outstanding source of nutrients with significant pharmaceutical value. Cultivation of this mushroom could be exploited to remediate soils and waters while simultaneously increasing grower incomes through the development of a circular economy.

Agricultural cropping systems that incorporate $S$. rugosoannulata tend to feature increased primary productivity and crop diversity of certain agricultural land types, enhancing the efficiency of reusable agricultural wastes. In the future, researchers should explore the myriad important roles this mushroom could play in sustainable cropping systems, particularly as farmers increasingly recognize its value as a promising component of modern agricultural practices.

\section{Acknowledgement}

This work was financed by the Key Research Project, Agroforestry System for Restoration and Bio-industry Technology Development (grant number 2017YFC0505101), Ministry of Sciences and Technology of China (grant number 2017YFC0505100); 'High-End Foreign Experts' in the High-Level Talent Recruitment Plan of Yunnan Province, 2021; National Sciences Foundation, China (NSFC) (grant number 41771063); and Thailand Science Research and Innovation grant 'Macrofungi diversity research from the Lancang-Mekong Watershed and surrounding areas' (grant number DBG6280009). Austin G. Smith at World Agroforestry (ICRAF), Kunming Institute of Botany, China, is also thanked for English editing. We thank the two anonymous reviewers for their corrections and suggestions to improve our manuscript.

\section{References}

Anasonye F, Winquist E, Kluczek-Turpeinen B, Räsänen M et al. 2014 - Fungal enzyme production and biodegradation of polychlorinated dibenzo-p-dioxins and dibenzofurans in contaminated sawmill soil. Chemosphere 110, 85-90.

Anasonye F, Winquist E, Räsänen M, Kontro J et al. 2015 - Bioremediation of TNT contaminated soil with fungi under laboratory and pilot scale conditions. International Biodeterioration \& Biodegradation 105, 7-12.

Bao R. 2015 - High yield of Stropharia rugosoannulata. Northwest A \& F University. Master Thesis.

Beluhan S, Ranogajec A. 2011 - Chemical composition and non-volatile components of Croatian wild edible mushrooms. Food chemistry 124(3), 1076-1082. 
Bruhn JN, Abright N, Mihail JD. 2010 - Forest farming of wine-cap Stropharia mushrooms. Agroforestry systems 79(2), 267-275.

Carneiro AA, Ferreira IC, Dueñas M, Barros L et al. 2013 - Chemical composition and antioxidant activity of dried powder formulations of Agaricus blazei and Lentinus edodes. Food Chemistry 138(4), 2168-2173.

Castellet-Rovira F, Lucas D, Villagrasa M, Rodríguez-Mozaz S et al. 2018 - Stropharia rugosoannulata and Gymnopilus luteofolius: Promising fungal species for pharmaceutical biodegradation in contaminated water. Journal of environmental management 207, 396-404.

CEMBN: Global mushroom business platform. 2019 - Available from: http://en.mushroommarket.net/ (Accessed on July 28, 2021).

Chen JC, Shen HS, Li YB, Xiao SG et al. 2010 - The effect of different culture mediums on the production and quality of Stropharia rugosoannulata. Edible Fungi of China 29(3), 18-19.

Demirbaş A. 2001 - Concentrations of 21 metals in 18 species of mushrooms growing in the East Black Sea region. Food Chemistry 75(4), 453-457.

de Oliveira JBH, Pereira PRC, dos Santos VS, Ferreira JM et al. 2020 - Stropharia. In: Amaresan N, Kumar MS, Annapurna K, Kumar K et al. Beneficial microbes in agro-ecology: bacteria and fungi. New York: Academic Press, Pp. 749-755.

Domondon D, Poppe J, Griensven L. 2000 - Fruit optimization with wastes used for outdoor cultivation of king Stropharia. Neophilologus 79(4), 619-628.

Duan L, Gan Y, Zhang W, Luo Y et al. 2018 - Planting trials of undergrowth Stropharia rugosoannulata. Journal of Southwest Forestry University 38(4), 206-209.

Duan L, Gan Y, Zhou B, Jing H et al. 2019 - Effect of Stropharia rugosoannulata under-forest cultivation on soil. Forest Inventory and Planning 44(1), 58-63.

Gong S, Zhao SF, Nie Y, Liu SC et al. 2016 - Economic benefits of Stropharia rugosoannulata under different cultivation methods and conditions. Edible Fungi of China 35(4), 35-38.

Gong S, Chen C, Zhu J, Qi G et al. 2018 - Effects of wine-cap Stropharia cultivation on soil nutrients and bacterial communities in forestlands of northern China. PeerJ 6, e5741.

Guo JM, Bai HL, Zhang H, Wang BS et al. 2010 - Determination of seven trace elements in Yunnan wild Lentinus edodes (Berk.) sing by atomic absorption spectrometry. Food Science and Technology 35(2), 228-230.

Hanafi FHM, Rezania S, Taib SM, Din MFM et al. 2018 - Environmentally sustainable applications of agro-based spent mushroom substrate (SMS): an overview. Journal of Material Cycles and Waste Management 20(3), 1383-1396.

Heleno SA, Ferreira RC, Antonio AL, Queiroz MJR et al. 2015 - Nutritional value, bioactive compounds and antioxidant properties of three edible mushrooms from Poland. Food bioscience 11, 48-55.

He P, Geng L, Wang J, Xu C. 2012 - Production, purification, molecular characterization and bioactivities of exopolysaccharides produced by the wine cap culinary-medicinal mushroom, Stropharia rugosoannulata 2\# (higher basidiomycetes). International Journal of Medicinal Mushrooms 14(4), 365-376.

Hu Q, Zhang R. 2013 - Recycling mode of fungi industry can promote efficiently utilization of agricultural wastes. Chinese Journal of Agricultural Resources and Regional Planning 34(6), $113-119$.

Hua F, Xing WL, Li WP, Huo J et al. 2021 - Determining the optimum cultivation time of Stropharia rugosoannulata according to the temperature conditions in Hebei Province. Edible Fungi of China 40(4), 18-22.

Huang JX, Yuan SN, Pan J, Zheng DH et al. 2019 - Yield of Stropharia rugosoannulata planted under rubber plantation and analysis of its quality of sporophore. Chinese Journal of Tropical Crops 40(1), 18-23.

Hyde KD, Xu J, Rapior S, Jeewon R et al. 2019 - The amazing potential of fungi: 50 ways we can exploit fungi industrially. Fungal Diversity 97(1), 1-136. 
Jedidi IK, Ayoub IK, Philippe T, Bouzouita N. 2017 - Chemical composition and nutritional value of three Tunisian wild edible mushrooms. Journal of Food Measurement and Characterization 11(4), 2069-2075.

Jiang K, Zhang JB, Liu QM, Xiao MP et al. 2017 - Research summary on edible fungi cultivation with rice chaff as main component. Edible Fungi of China 36(3), 1-6.

Jiang L, Hou Y, Ding X. 2020 - Structure identification and biological activities of a new polysaccharides from Stropharia rugosoannulata. Latin American Journal of Pharmacy 39(8), 1594-1604.

Jin Z, Li Y, Ren J, Qin N. 2018 - Yield, nutritional content, and antioxidant activity of Pleurotus ostreatus on corncobs supplemented with herb residues. Mycobiology 46(1), 24-32.

Jin Z, Wu H, Jiang J, Xu Y et al. 2020 - Field experiment on uncooked cultivation of Stropharia rugosoannulata in mountainous areas of southern Zhejiang province. Hans Journal of Agricultural Sciences 10(1), 26-34.

Li DY, Wei GJ, Qin ZS, Huang YD et al. 2012 - Stropharia rugosoannulata cultivation technique in small arch shed with mulberry sawdust. Southern Horticulture 23(1), 48-49.

Li ZW, Han HD, Chen MJ, Lin ZN et al. 2013 - Effects of intercropping Stropharia rugosoannulata on tea garden soil and tea growth. Fujian Journal of Agricultural Sciences 28(11), 1088-1092.

Liu YT, Sun J, Luo ZY, Rao SQ et al. 2012 - Chemical composition of five wild edible mushrooms collected from Southwest China and their antihyperglycemic and antioxidant activity. Food and Chemical Toxicology 50(5), 1238-1244.

Liu D, Chen YQ, Xiao XW, Zhong RT et al. 2019a - Nutrient properties and nuclear magnetic resonance-based metabonomic analysis of macrofungi. Foods 8(9), 397.

Liu JL, Tang L, Chen S, Dong YP et al. 2019b - Optimization of carbon and nitrogen sources and culture conditions of liquid culture medium of Stropharia rugosoannulata. Heilongjiang Agricultural Sciences 4, 94-99.

Liu Y, Hu CF, Feng X, Cheng L et al. 2020 - Isolation, characterization and antioxidant of polysaccharides from Stropharia rugosoannulata. International Journal of Biological Macromolecules 155, 883-889.

Luo H, Li X, Li G, Pan Y et al. 2006 - Acanthocytes of Stropharia rugosoannulata function as a nematode-attacking device. Applied and environmental microbiology 72(4), 2982-2987.

Mallikarjuna SE, Ranjini A, Haware DJ, Vijayalakshmi MR et al. 2012 - Mineral composition of four edible mushrooms. Journal of Chemistry 2013, 1-5.

Meyer V, Basenko EY, Benz JP, Braus GH et al. 2020 - Growing a circular economy with fungal biotechnology: a white paper. Fungal biology and biotechnology 7(5), 1-23.

Nie Y, Liu JX, Zhao C, Li LJ et al. 2016 - Economic benefit analysis of interplanting different edible fungi with corns. Edible Fungi of China 35(2), 43-45.

Owaid MN. 2015 - Mineral elements content in two sources of Agaricus bisporus in Iraqi market. Journal of Advanced \& Applied Sciences 3(2), 46-50.

Pegler DN. 2001 - Useful fungi of the world: Wine cap - The gardener's mushroom. Mycologist 2(15), 50-51.

Pozdnyakova N, Schlosser D, Dubrovskaya E, Balandina S et al. 2018 - The degradative activity and adaptation potential of the litter-decomposing fungus Stropharia rugosoannulata. World Journal of Microbiology and Biotechnology 34(9), 1-14.

Raskin I. 1992 - Role of salicylic acid in plants. Annual review of plant biology 43(1), 439-463.

Sharma VP, Sharma SR, Kumar S. 2007 - Cultivation of least-exploited commercial mushrooms. Mushroom Biology and Biotechnology 213, 167-192.

She DF, Fan WG, Xu YJ, Liu PT et al. 2007 - Progress of cultivation technology of Stropharia rugosoannulata. Seed 26(1), 84-87

Stamets P, Chilton JS. 1983 - The mushroom cultivator. Agarikon Press, Washington.

Stamets P. 2011 - Growing gourmet and medicinal mushrooms. Ten Speed Press, Berkeley. 
Steffen KT, Schubert S, Tuomela M, Hatakka A et al. 2007 - Enhancement of bioconversion of high-molecular mass polycyclic aromatic hydrocarbons in contaminated non-sterile soil by litter-decomposing fungi. Biodegradation 18(3), 359-369.

Stojković D, Reis FS, Glamočlija J, Ćirić A et al. 2014 - Cultivated strains of Agaricus bisporus and $A$. brasiliensis: chemical characterization and evaluation of antioxidant and antimicrobial properties for the final healthy product-natural preservatives in yoghurt. Food \& function 5(7), 1602-1612.

Sun M. 2013 - Mycelium culture and the variation regularity of extracellular enzyme activity of Stropharia rugosoannulata Farlow. YanBian University. Master Thesis.

Suzuki T, Ono A, Choi JH, Wu J et al. 2019 - The complete mitochondrial genome sequence of the edible mushroom Stropharia rugosoannulata (Strophariaceae, Basidiomycota). Mitochondrial DNA Part B 4(1), 570-572.

Szudyga K. 1978 - Stropharia rugosoannulata. In: Chang ST, Hayes WA (eds). The biology and cultivation of edible mushrooms. Orlando: Academic Press, Pp. 559-572.

Tian GT, Xu XZ, Zhang KY. 2002 - High yield cultivation of Stropharia rugosoannulata in tableland area. Edible Fungi of China 21(3), 9-16.

Valentín L, Oesch-Kuisma H, Steffen KT, Kähkönen MA et al. 2013 - Mycoremediation of wood and soil from an old sawmill area contaminated for decades. Journal of hazardous materials 260, 668-675.

Wang XW. 2007 - Nutrition components analyse, extraction and antioxidant properties of polysaccharide of Stropharia rugosoannulata. Nanjing Normal University. Master Thesis.

Wang SH, Li YS. 2008 - The optimization of carbonaceous compounds and nitrogenous substances for liquid culture media of Stropharia rugosoannulata. Northern Horticulture 5, 219-221.

Wang H, Chen H, Zhang JJ, Chen MJ. 2018 - Research progresses on bioactive components in Stropharia rugosoannulata and their pharmacological effects. Acta Edulis Fungi 25(04), 115-120.

Wan S, Bau T. 2005 - Advances in the study on Stropharia rugosoannulata. Acta Edulis Fungi 12(4), 57-64.

Wisitrassameewong K, Karunarathna SC, Thongklang N, Zhao R et al. 2012 - Agaricus subrufescens: a review. Saudi journal of biological sciences 19(2), 131-146.

Wu J, Fushimi K, Tokuyama S, Ohno M et al. 2011 - Functional-food constituents in the fruiting bodies of Stropharia rugosoannulata. Bioscience, biotechnology, and biochemistry 75(8), $1631-1634$.

Wu J, Tokuyama S, Nagai K, Yasuda N et al. 2012 - Strophasterols A to D with an unprecedented steroid skeleton: from the mushroom Stropharia rugosoannulata. Angewandte chemie 124(43), 10978-10980.

Wu J, Kobori H, Kawaide M, Suzuki T et al. 2013a - Isolation of bioactive steroids from the Stropharia rugosoannulata mushroom and absolute configuration of strophasterol B. Bioscience, biotechnology, and biochemistry 77(8), 1779-1781.

Wu J, Suzuki T, Choi JH, Yasuda N et al. 2013b - An unusual sterol from the mushroom Stropharia rugosoannulata. Tetrahedron Letters 54(36), 4900-4902.

Yan PS, Li GF, Jiang JH, Zhang YC et al. 2001 - Effects of nutrients and environmental factors on the mycelial growth of Stropharia rugosoannulata. Acta Edulis Fungi 8(1), 5-9.

Yan PS, Jiang JH. 2005 - Preliminary research of the RAPD molecular marker-assisted breeding of the edible basidiomycete Stropharia rugosoannulata. World Journal of Microbiology and Biotechnology 21(4), 559-563.

Yan QX, Huang MX, Sun P, Cheng SX et al. 2020 - Steroids, fatty acids and ceramide from the mushroom Stropharia rugosoannulata Farlow apud Murrill. Biochemical Systematics and Ecology 88, 103963. 
Yang Y, Li C, Ni S, Zhang H et al. 2021 - Ultrastructure and development of acanthocytes, specialized cells in Stropharia rugosoannulata, revealed by scanning electron microscopy (SEM) and cryo-SEM. Mycologia 113(1), 65-77.

Yu P, Sun M, Fu C, Chen Y et al. 2014 - Extracellular enzyme production by Stropharia rugosoannulata Farlow during cultivation on raw materials. Edible Fungi of China 33(1), 4850.

Zhai X, Zhao A, Geng L, Xu C. 2013 - Fermentation characteristics and hypoglycemic activity of an exopolysaccharide produced by submerged culture of Stropharia rugosoannulata\# 2. Annals of Microbiology 63(3), 1013-1020.

Zhou B, Jia L, Meng F, Song Z et al. 2010 - Statistical optimization of cultivation conditions for exopolysacchride production and mycelia growth by Stropharia rugosoannulata. Annals of microbiology 60(1), 89-96.

Zhang JJ, Li Y, Zhou T, Xu DP et al. 2016 - Bioactivities and health benefits of mushrooms mainly from China. Molecules 21(7), 938.

Zhang P, Wang YF, Shi L, Wang JH et al. 2017 - Technology research on cultivation of Stropharia rugosoannulata using Auricularia heimuer residue. Edible Fungi of China 36(1), 32-35.

Zhang SM, He JL, Qiu LY, Liu Y et al. 2005 - Effect of different carbon nitrogen nutrition sources and temperature on mycelial growth of Stropharia rugosoannulata. Journal of microbiology 25(6), 32-34.

Zhang W, Tian G, Geng X, Zhao Y et al. 2014 - Isolation and characterization of a novel lectin from the edible mushroom Stropharia rugosoannulata. Molecules 19(12), 19880-19891.

Zhu JX. 2018 - Breeding of thermotolerant strains of Stropharia rugosoannulata. Shandong Agricultural University. Master Thesis. 